\title{
DETERMINAÇÃO DE MARCADORES DE ENVELHECIMENTO EM CACHAÇAS
}

\author{
Francisco W. B. AQUINO ${ }^{2}$, Ronaldo F. NASCIMENTO ${ }^{3}$, \\ Sueli RODRIGUES ${ }^{2, *}$, Antônio Renato S. CASEMIRO ${ }^{2}$
}

\section{RESUMO}

O envelhecimento de bebidas em tonéis de madeira é responsável pela melhoria do sabor e do aroma do produto. Embora o carvalho seja a madeira tradicionalmente utilizada para envelhecimento de bebidas, no Brasil é comum o uso de outras madeiras, como o bálsamo, e madeiras regionais utilizadas por pequenos produtores, fazendo com que o tipo de tonel usado para o envelhecimento possa variar muito. Neste trabalho, é apresentado um estudo da concentração de furanos e fenólicos de baixo peso molecular (ácido gálico, 5-hidroximetilfurfural, furfural, ácido vanílico, ácido siríngico, vanilina, siringaldeído, coniferaldeído, sinapaldeído e cumarina), considerados marcadores de envelhecimento, em cachaças oriundas de pequenos produtores das cinco regiões fabricantes de cachaça do Estado do Ceará. Os resultados obtidos foram comparados com cachaças comerciais consideradas produtos de primeira linha, produzidas nos Estados de Pernambuco, Ceará e Minas Gerais, e apresentaram boa concordância.

Palavras-chave: cachaça, bebidas envelhecidas, fenólicos, HPLC, marcadores de envelhecimento.

\section{SUMMARY}

DETERMINATION OF AGING MARKS IN SUGAR CANE SPIRITS. Alcoholic beverages are usually aged in wooden barrels. This technique improves the taste and the aroma of the product. Although oak is the main wood employed to age beverages, other woods as Balm and local woods are largely employed in Brazil, specially by sugar cane spirits small producers. In this work is presented a study of furans and low molecular weight phenolic compounds (gallic acid, 5-hydroxymethylfurufural, furfural, vanillic acid, syringic acid, vanillin, syringaldehyde, coniferaldehyde, sinapaldehyde and coumarin), considered as aging markers in aged sugar cane spirits from small producers of Ceará State (Brazil). The obtained results were compared to reference samples of for export sugar cane spirits and presented good agreement.

Keywords: sugar cane spirit, aged beverages, phenolics, HPLC, aging markers.

\section{1- INTRODUÇÃO}

Definida, oficialmente, como a denominação típica e exclusiva da aguardente de cana produzida no Brasil, com graduação alcoólica de 38 a $48 \%$ em volume, a $20^{\circ} \mathrm{C}$ e com características sensoriais peculiares [1], a cachaça, com sua origem datando do século XVII, quando a cana-de-açúcar começou a ser plantada na Região Nordeste em meio à colonização portuguesa [2], é bebida tradicional do Brasil. Da mesma maneira, acontece em outros países do mundo, onde também existem bebidas que, em muitos casos, chegam até mesmo a fazer parte das suas tradições.

A produção de cachaça pode ser divida em quatro etapas principais: obtenção do mosto, fermentação, destilação e envelhecimento. Por melhor que sejam realizadas as três primeiras etapas do processo produtivo, o destilado recém-

${ }^{1}$ Recebido para publicação em 11/2/2005. Aceito para publicação em 23/1/2006 (001483)

2Departamento de Tecnologia de Alimentos. Universidade Federal do Ceará

Avenida Mister Hull, 2.977, Bloco 858

CEP 60356-000 - Fortaleza (CE), Brasil

E-mail:sueli@ufc.br

3Departamento de Físico-Química. Universidade Federal do Ceará

Avenida Mister Hull, $n^{\circ}$ 2.977, Bloco 940

CEP 60356-000 - Fortaleza (CE), Brasil

*A quem a correspondência deve ser enviada obtido apresentará sabor seco, ardente e um aroma não muito agradável [3-7]. Dessa forma, o envelhecimento se torna fundamental para que a cachaça adquira as características desejadas pelos seus consumidores, pois ao longo deste período a bebida adquire os atributos necessários de cor, aroma e sabor típicos dos destilados de alta qualidade [8-10].

A literatura internacional, principalmente a referente ao uísque e ao vinho, aponta vários compostos que podem ser utilizados como marcadores de envelhecimento em bebidas. Entre eles, temos os compostos fenólicos de baixo peso molecular, que são extraídos da madeira durante o seu período de guarda por vários mecanismos de degradação da celulose, hemicelulose e da lignina, que se constituem nos componentes majoritários da madeira [11-14].

Durante o envelhecimento são gerados os congêneres que agregam valores sensoriais e, conseqüentemente, financeiros aos destilados. Em função das peculiaridades existentes no processo de envelhecimento, como: os aspectos da tanoaria (capacidade do recipiente, tipo de madeira, intensidade da sua queima, número de virtudes do tonel, etc.), os aspectos ambientais (temperatura e umidade) e o tempo de guarda propriamente dito; um destilado pode ser detentor de características singulares que o valorize e o destaque em relação aos demais.

Embora a madeira tradicional utilizada para o envelhecimento de bebidas seja o carvalho, no Brasil é comum 
o uso de bálsamo e até mesmo de madeiras regionais para construção de tonéis, devido ao alto custo do carvalho, que é uma madeira típica do Hemisfério Norte. Estima-se que o Brasil possua cerca de 30 mil produtores de cachaça, sendo a maioria deles pequenos produtores. Isso pode significar que uma infinidade de madeiras seja utilizada para o envelhecimento da bebida.

Apesar de a qualidade superior de bebidas envelhecidas ser de conhecimento geral, existem poucos trabalhos relacionados à análise dos compostos fenólicos de baixo peso molecular (considerados como marcadores de envelhecimento). Neste trabalho, é apresentado um estudo do perfil destes compostos em cachaças envelhecidas obtidas de pequenos produtores das cinco regiões produtoras do Estado do Ceará: Serra do Ibiapaba, Maciço do Baturité, Região Metropolitana de Fortaleza, Vale do Cariri e Sertão Central. Os resultados obtidos foram comparados aos de cachaças comerciais consideradas produtos de primeira linha, produzidas nos Estados de Pernambuco, Ceará e Minas Gerais, e apresentaram boa concordância.

\section{2- MATERIAL E MÉTODOS}

\section{1 - Obtenção das amostras de cachaça}

Todas as cachaças usadas no trabalho pertencem ao acervo do Laboratório de Biotecnologia (Labiotec/Detal/ UFC). A autenticidade do envelhecimento das cachaças foi comprovada, pois as amostras foram adquiridas dos produtores por membros do laboratório. Uma cachaça mineira comercial envelhecida, comercializada em todo o Brasil por uma grande rede de supermercados; uma cachaça pernambucana comercial envelhecida de grande vendagem; e uma amostra 'top de linha' envelhecida do maior fabricante e exportador do Estado do Ceará; foram empregadas para efeito comparativo.

\section{2 - Padrões cromatográficos}

Os padrões utilizados neste trabalho: ácido gálico, 5-hidroximetilfurfural, furfural, ácido vanílico, ácido siríngico, vanilina, siringaldeído, coniferaldeído, sinapaldeído e cumarina com pureza $>99 \%$ foram obtidos das companhias Sigma-Aldrich (Saint Louis, MO - EUA) e Acros Organics (New Jersey, EUA).

Os furanos 5-hidroximetilfurfural e furfural, embora não sendo originados da degradação da lignina ou da hemicelulose, foram incluídos no estudo por estarem associados à degradação térmica de pentoses e hexoses respectivamente, provenientes do tratamento térmico da madeira, sendo extraídos e incorporados à bebida [3, 15, 16].

\section{3 - Solventes}

Na composição da fase móvel foi empregado metanol, grau de pureza cromatográfico, da Tedia Company Inc. (Fairfield, OH - EUA), água ultrapura obtida por sistema de osmose reversa e filtrada em membranas de ultrafiltração de 0,45 $\mu \mathrm{m}$, da Fresenius Kabi Brasil (Aquiraz, CE - BRA) e ácido acético PA. Merck (Darmstadt, GER).

\section{4 - Equipamentos}

Todas as análises foram efetuadas em um sistema cromatográfico de alta eficiência $\operatorname{Varian}^{\circledR}$, gerenciado pelo software de controle e aquisição de dados Star WS, versão 5.5, e composto por duas bombas de alta pressão Pro Star 210; um forno para coluna modelo Timbeline 101; um detector UV-Vis Pro Star de duplo canal, com variação programável de comprimento de onda durante as análises, modelo 345 e injetor Rheodyne com loop de $20 \mu \mathrm{L}$.

Os componentes das amostras e padrões foram separados por uma coluna Merck LiChrospher 100 RP-18 endcapped, de $250 \mathrm{~mm}$ x $4 \mathrm{~mm}$ (comprimento x diâmetro interno) empacotada com partículas totalmente esféricas de $5 \mu \mathrm{m}$. Antes de serem cromatografadas as amostras e padrões foram filtrados em um sistema Hamilton aclopável a seringas, composto por um pré-filtro de microfibras de borossilicato e por uma membrana filtrante de nylon com abertura de 0,45 $\mu \mathrm{m}$, ambos com 0,13 cm de diâmetro.

\section{5 - Condições cromatográficas}

As análises foram realizadas por meio da injeção direta das amostras previamente filtradas conforme descrito no Item 2.4. Quando algum dos analitos da amostra exibiu concentrações acima do limite superior da sua respectiva curva de calibração, a referida amostra foi diluída com uma solução etanólica a 50\% e reinjetada. Os analitos foram identificados através do tempo de retenção e da dopagem de uma amostra com padrões autênticos.

Foram utilizadas duas fases móveis compostas de água/ácido acético 98:2 (v/v) e metanol/água/ácido acético 70:28:2 (v/v/v) com fluxo total de 1,25 mL, coluna termostatizada em $40^{\circ} \mathrm{C}$ e comprimento de onda variável de acordo com as condições cromatográficas apresentadas na Tabela 1. O método empregado foi previamente validado, tendo apresentado boa linearidade, seletividade, precisão e exatidão [17].

TABELA 1 - Condições cromatográficas utilizadas

\begin{tabular}{lcccc}
\hline $\begin{array}{l}\text { Tempo } \\
(\boldsymbol{m i n})\end{array}$ & $\begin{array}{c}\text { Solvente } \boldsymbol{A} \\
(\%, \boldsymbol{v} / \mathbf{v})\end{array}$ & $\begin{array}{c}\text { Solvente } \boldsymbol{B} \\
(\%, \boldsymbol{v} / \mathbf{v})\end{array}$ & $\begin{array}{c}\text { Fluxo total } \\
(\mathbf{m L} / \mathbf{m i n})\end{array}$ & $\begin{array}{c}\text { Comprimento } \\
\mathbf{d e} \text { onda } \\
(\mathbf{n m})\end{array}$ \\
\hline 0 & 100 & 0 & 1,25 & 271 \\
3 & 100 & 0 & 1,25 & 271 \\
6 & 100 & 0 & 1,25 & 280 \\
25 & 60 & 40 & 1,25 & 345 \\
28 & 60 & 40 & 1,25 & 320 \\
43 & 40 & 60 & 1,25 & 271 \\
50 & 0 & 100 & 1,25 & 271 \\
55 & 100 & 0 & 1,25 & 271 \\
60 & 100 & 0 & 1,25 & 271 \\
\hline Solvente A - água:ácido acético, $98: 2 \%(\mathrm{v} / \mathrm{v})$. Solvente B - metanol:água:ácido acético, \\
$70 \cdot 28 \%$ (v/v/v)
\end{tabular}
$70: 28: 2 \%(\mathrm{v} / \mathrm{v} / \mathrm{v})$ 


\section{3 - RESULTADOS E DISCUSSÃO}

Ao todo foram analisadas 28 amostras de cachaças envelhecidas de pequenos produtores do Estado do Ceará e três amostras de cachaças de primeira linha de grandes produtores dos Estados de Minas Gerais, Pernambuco e Ceará. Os resultados obtidos são apresentados na Tabela 2.

Para os resultados apresentados na Tabela 2 foram encontrados os seguintes coeficientes de variação por analito: ácido gálico, 19,94\%; 5-hidroximetilfurfural, 6,59\%; furfural, 6,25\%; ácido vanílico, 3,71\%; ácido siríngico, 4,58\%; vanilina, 4,95\%; siringaldeído, $6,35 \%$; coniferaldeído, 4,75\%; sinapaldeído, 5,08\%; cumarina, 10,15\%.

Apesar de elevados, os valores para o ácido gálico e para a cumarina podem ser justificados pelos baixos níveis em que são encontrados nas amostras, nas quais o ácido gálico varias vezes apresentou-se abaixo do limite de deteç̧ão e para cumarina, mesmo tendo sido detectados com pequena possibilidade de equívoco (devido às características do método validado) seus valores em algumas amostras foram calculados com incremento do erro analítico devido às suas áreas correspondentes encontrarem-se entre os limites de detecção e de quantificação.

Com relação ao 5-HMF, mesmo com seu coeficiente de variação figurando em um nível relativamente baixo, a remoção dos valores quantificados nas amostras Cariri 9, Maciço de Baturité 1 e Ibiapaba 1, devido a estas amostras apresentarem teores de 5-HMF anomalamente elevados quando comparados aos determinados nas demais amostras (Tabela 2), reduziu o valor do coeficiente de variação em aproximadamente três vezes (de 6,59; para 2,17\%). Altos teores de 5-HMF são um indicativo de condições anômalas no envelhecimento das bebidas.

Para os demais analitos, as suas variações, quer em termos absolutos ou na forma de coeficiente de variação, e ainda com relação às proporções entre si, podem ser consideradas como normais devido, principalmente, às diferentes madeiras dos seus tonéis e ao seu tempo de guarda.

TABELA 2 - Quantificação (ppm) de compostos fenólicos de baixo peso molecular, em cachaças envelhecidas do Ceará

\begin{tabular}{|c|c|c|c|c|c|c|c|c|c|c|c|}
\hline Região & $\begin{array}{l}\text { Ácido } \\
\text { gálico }\end{array}$ & 5-HMF & Furfural & $\begin{array}{c}\text { Ácido } \\
\text { vanílico }\end{array}$ & $\begin{array}{c}\text { Ácido } \\
\text { Siríngico }\end{array}$ & Vanilina & Siringaldeído & Coniferaldeído & Sinapaldeído & Cumarina & Total \\
\hline Cariri 1 & $<\mathrm{LD}$ & 7,8059 & 1,2230 & 0,5646 & 1,0311 & 0,5204 & 6,5633 & 0,3337 & 0,3385 & $<L D$ & 18,3805 \\
\hline Cariri 2 & $<\mathrm{LD}$ & 0,3789 & 0,2790 & 0,4786 & 0,3772 & 0,1059 & 0,1405 & 0,1556 & 0,4512 & $<\mathrm{LD}$ & 2,3669 \\
\hline Cariri 3 & $<\mathrm{LD}$ & $<\mathrm{LQ}$ & 0,5941 & 0,1592 & 0,2261 & 0,1545 & 0,2478 & $<\mathrm{LD}$ & 5,1228 & $0,0694^{*}$ & 6,5045 \\
\hline Cariri 4 & 0,3561 & 0,3290 & 2,2803 & 2,8772 & 7,5500 & 5,8665 & 32,3258 & 1,4172 & 0,9505 & $0,051^{*}$ & 53,9526 \\
\hline Cariri 5 & 1,7580 & 1,0510 & 0,9133 & 1,7086 & 4,9019 & 6,6682 & 37,3563 & 1,8104 & 0,7713 & $0,0457^{*}$ & 56,9390 \\
\hline Cariri 6 & 0,4427 & 0,3568 & 9,6183 & 0,1665 & 0,6189 & 0,4538 & 0,1280 & $<\mathrm{LD}$ & 4,0970 & $0,0532^{*}$ & 15,8820 \\
\hline Cariri 7 & $<\mathrm{LD}$ & 4,8790 & 0,2442 & 0,7015 & $<\mathrm{LD}$ & $<\mathrm{LD}$ & 9,2634 & 0,0850 & 0,5379 & $0,0571^{*}$ & 15,7110 \\
\hline Cariri 8 & $<\mathrm{LD}$ & 2,7906 & 0,4576 & 0,3853 & 1,3832 & 0,1351 & 0,0080 & 0,0014 & 0,3294 & $0,0582^{*}$ & 5,4906 \\
\hline Cariri 9 & $<\mathrm{LD}$ & 10,1796 & 0,3043 & $<\mathrm{LD}$ & 0,4117 & 0,1582 & 1,3358 & 0,0162 & 0,2725 & $0,0502^{*}$ & 12,6783 \\
\hline Cariri 10 & 1,2640 & 0,3114 & 0,2541 & 0,5315 & 0,1258 & 0,6096 & 1,9076 & 0,6254 & 0,8872 & $0,0321^{*}$ & 6,5166 \\
\hline M. Baturité 1 & $<\mathrm{LD}$ & 21,1840 & 1,0413 & 0,2340 & 1,1231 & 1,8155 & 10,5564 & 0,0499 & 1,6384 & 0,3788 & 38,0214 \\
\hline M. Baturité 2 & 2,6480 & 0,7585 & 0,6956 & 2,3104 & 1,1434 & 1,5193 & 1,9934 & 1,6567 & 0,3918 & $0,0569^{*}$ & 13,1171 \\
\hline M. Baturité 3 & $<\mathrm{LD}$ & $<\mathrm{LQ}$ & 0,2555 & 0,5969 & 0,7341 & 0,5214 & 0,4678 & $<\mathrm{LQ}$ & 4,6884 & $0,083^{*}$ & 7,2641 \\
\hline M. Baturité 4 & $<\mathrm{LD}$ & 0,1812 & 0,6260 & 1,4716 & 4,8728 & 4,4535 & 2,5199 & 1,1647 & 0,3790 & 0,9747 & 16,6434 \\
\hline M. Baturité 5 & $<\mathrm{LD}$ & 0,0578 & 0,1576 & 1,8684 & 3,2546 & 2,3480 & 7,6857 & 1,4656 & 0,2684 & $0,0486^{*}$ & 17,1061 \\
\hline M. Baturité 6 & 0,2087 & 0,4631 & 0,1959 & 1,0613 & 8,9574 & 0,1832 & 2,2806 & $<\mathrm{LD}$ & 6,1073 & $0,0859^{*}$ & 19,4575 \\
\hline M. Baturité 7 & $<\mathrm{LD}$ & 1,0707 & 1,5428 & 3,5375 & 6,1898 & 4,3442 & 13,2293 & 1,5669 & 0,7502 & $0,0703^{*}$ & 32,2314 \\
\hline M. Baturité 8 & 0,3096 & 0,4599 & 0,2296 & 2,1351 & 7,8195 & 0,2156 & 1,3320 & $<\mathrm{LD}$ & 8,1264 & $0,0672^{*}$ & 20,6277 \\
\hline M. Baturité 9 & $<L D$ & 0,6450 & 0,1698 & 0,3092 & 0,6902 & 0,5681 & 0,4281 & 0,1062 & 0,5518 & 0,4116 & 3,8800 \\
\hline Sertão 1 & $<$ LD & 1,18110 & 0,2165 & 0,0971 & 0,3670 & 0,2291 & 5,2510 & 0,2024 & 0,7354 & 0,1892 & 9,0987 \\
\hline Sertão 2 & $<L D$ & 0,4123 & 1,4953 & $<\mathrm{LD}$ & $<\mathrm{LD}$ & 0,1465 & $<L D$ & 0,1609 & 0,2654 & $<L D$ & 2,4804 \\
\hline RMF 1 & $<\mathrm{LD}$ & 2,0563 & 0,3261 & 0,2912 & 0,1276 & 0,3389 & 0,4673 & 1,3694 & 0,1580 & $0,0561^{*}$ & 5,1348 \\
\hline RMF 2 & $<L D$ & 5,4015 & 0,3312 & 0,7870 & 2,7502 & 2,1484 & 4,9885 & 0,1714 & 0,6205 & 0,3568 & 17,5555 \\
\hline RMF 4 & $<\mathrm{LD}$ & LD & 0,1822 & 0,3224 & 0,1581 & 0,5322 & 0,4129 & $<L Q$ & 0,9710 & 0,3562 & 2,9350 \\
\hline RMF 5 & $<\mathrm{LD}$ & LD & 0,1759 & 0,1302 & 0,7762 & 1,0955 & 2,6894 & $<L Q$ & 0,1809 & $0,0592^{*}$ & 5,0481 \\
\hline Ibiapaba 1 & 10,6324 & 19,0502 & 4,7810 & 1,7163 & 1,7943 & 0,7739 & 0,9678 & 0,3988 & 0,5353 & $<$ LD & 40,6500 \\
\hline Ibiapaba 2 & $<L D$ & 5,0377 & 1,3810 & 0,6013 & 0,6652 & 0,4253 & 2,2902 & 1,4666 & 0,6413 & $<\mathrm{LD}$ & 12,5086 \\
\hline Ibiapaba 3 & $<\mathrm{LD}$ & 0,4405 & 0,7389 & 0,2171 & 0,7957 & 0,6100 & $<L D$ & $<\mathrm{LD}$ & 0,2980 & $0,0797^{*}$ & 3,1002 \\
\hline Ref. 1 & $<L D$ & 1,8330 & 0,6927 & 0,9443 & 0,6579 & 1,4903 & 5,1776 & 1,2496 & 1,1889 & $<L Q$ & 13,2343 \\
\hline Ref. 2 & $<L D$ & 0,5791 & 1,1271 & 1,7113 & 1,3417 & 1,4984 & 1,6596 & 1,1847 & 2,0813 & 0,1287 & 11,3119 \\
\hline Ref. 3 & 0,4584 & 0,0585 & 0,4984 & 0,3269 & 3,8236 & 1,6350 & 11,9588 & 1,3262 & 0,4365 & $<L Q$ & 20,5223 \\
\hline
\end{tabular}

*Concentração em ppm para cumarina <LQ, sendo desta forma estimada matematicamente pelo software controlador do sistema cromatográfico 
A Figura 1 apresenta um comparativo entre os valores médios determinados para cada analito nas amostras cearenses envelhecidas e nas amostras comerciais de referência. A Figura 2 apresenta um comparativo entre o teor médio (ppm) dos compostos analisados nas amostras de cachaças envelhecidas do Ceará e nas cachaças de referência. Ambas as Figuras desconsideram os valores anômalos referentes às amostras Cariri 9, Maciço de Baturité 1 e Ibiapaba 1 (Tabela 2).

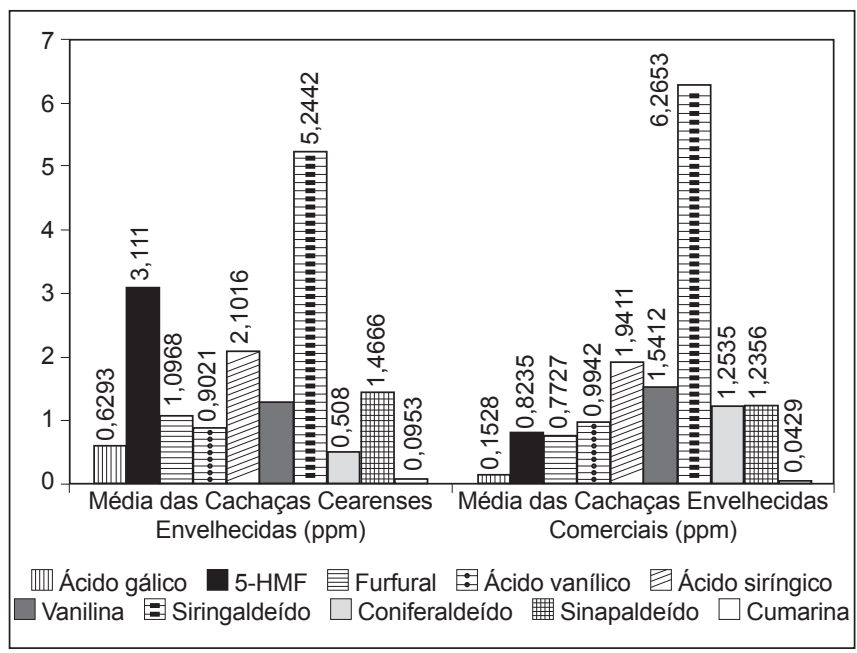

FIGURA 1 - Comparativo entre os valores médios (ppm) determinados para cada analito nas amostras de cachaças cearenses envelhecidas e nas cachaças envelhecidas de referência analisadas

Da esquerda para a direira: ácido gálico; 5-HMF; furfural, ácido vanílico, ácido siríngico, vanilina, siringaldeído, coniferaldeído, sinapaldeído, cumarina

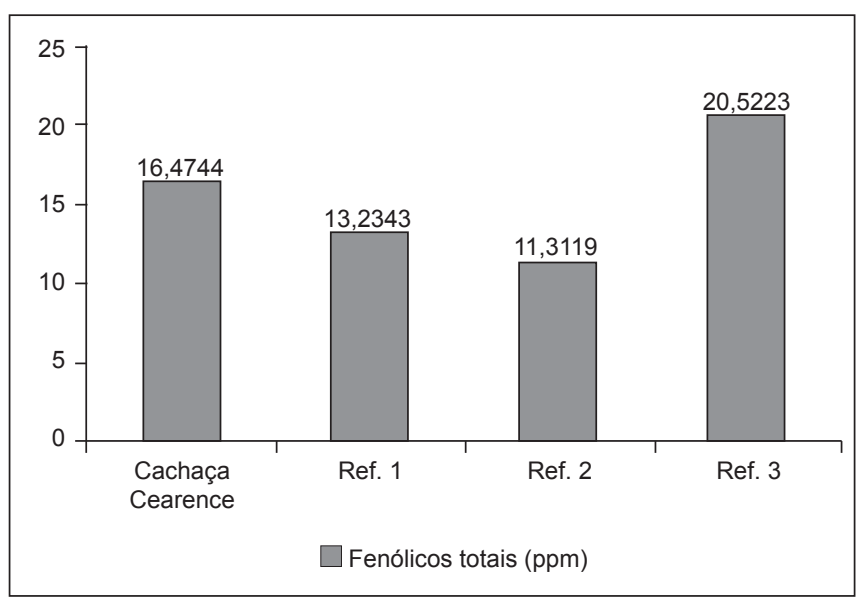

FIGURA 2 - Comparativo entre o teor médio dos compostos fenólicos (ppm), de baixo peso molecular, analisados nas amostras de cachaças envelhecidas do Ceará e nas cachaças de referência

Da esquerda para a direira: ácido gálico; 5-HMF; furfural, ácido vanílico, ácido siríngico, vanilina, siringaldeído, coniferaldeído, sinapaldeído, cumarina

No tocante à distribuição média dos analitos (Figura 1), com exceção para o 5-HMF, observa-se uma boa correspon- dência. O somatório dos teores individuais por analito para a média das amostras de cachaças cearenses analisadas, encontra-se em um patamar que figura entre as quantidades mínimas e máximas determinadas nas amostras de referência (Figura 2).

\section{4- CONCLUSÕES}

De acordo com o perfil dos compostos fenólicos de baixo peso molecular encontrados nas amostras de cachaça cearense, a média obtida para o total destes compostos, e ainda a sua distribuição, assemelharam-se intensamente com os mesmos dados gerados para a média das cachaças envelhecidas de referência.

As três cachaças com altos níveis de 5-HMF: Cariri 9, Maciço de Baturité 1 e Ibiapaba 1, podem ser oriundas de tonéis bastante usados, que não sofreram tratamento para a sua recuperação, como a raspagem de suas paredes e uma nova queima, uma vez que o tratamento térmico e a manutenção do tonel influenciam a composição química final do produto.

Embora não seja possível determinar, com precisão, o tempo de envelhecimento de uma bebida apenas com base na quantificação dos compostos fenólicos de baixo peso molecular, sua determinação pode ser utilizada como indicativo de sua autenticidade, uma vez que estes compostos não são encontrados em cachaças não envelhecidas. Esta análise é bastante útil no controle de qualidade da bebida, já que a legislação brasileira permite a adição de corante caramelo para correção da cor de cachaças envelhecidas. Entretanto, a adição deste corante em cachaças não envelhecidas pode levar o consumidor a crer que está adquirindo um produto envelhecido. Dessa forma, a presença ou ausência de marcadores de envelhecimento na bebida pode revelar à fraude ou atestar sua qualidade.

\section{5- REFERÊNCIAS BIBLIOGRAFICAS}

[1] BRASIL. Decreto ${ }^{\circ} 4.072$, de 21 de dezembro de 2002. Ministério da Agricultura. Diário Oficial da União, 3 jan. 2002.

[2] CARVAlHO, M. \& SILVA; P. SIlvestre. Cachaça: uma alegre história brasileira., São Paulo, Caninha 51, 157 p., 1988.

[3] BOZA, Y; OETTERER, M. Envelhecimento de aguardente de cana. Boletim da sbCTA., v. 33, n. 1, p. 8-15, 1999.

[4] CARDELLO, H.M.A.B.; FARIA, J.B. Análise da aceitação de aguardentes de cana por testes afetivos e mapa de preferência interno. Cienc. Tecnol. Aliment., v. 20, n. 1, p. 32-36, 2000.

[5] CERDÁN, T.G.; MOZAZ S.R.; AZPILICUETA, C.A. Volatile composition of aged wine in used barrels of French oak and of American oak. Food Res. Int., v. 35, p. 603-610, 2002.

[6] ESCALONA, H.; BIRKMYRE, L.; PIGGOTT, J.R.; PATERSON A. Effect of maturation in small oak casks on the volatility of red wine aroma compounds. Anal. Chim. Acta., n. 458, p. 45-54, 2002. 
[7] PLAZA, D.E.M.; REYERO, J.R.; PARDO, F.; SALINAS, M.R. Comparison of wine aromas with different tannic content aged in French oak barrels. Anal. Chim. Acta., n. 458, p. 139-145, 2002.

[8] MOSEDALE, J.R.; PUECH, J.L. Wood maturation of distilled beverages. Trends in Food Science \& Technology., v. 9, p. 95-101, 1998.

[9] CAMPOS, J.O.S. Emprego de extratos aromáticos de madeiras regionais como agentes de envelhecimento acelerado de aguardentes. Fortaleza, 2000, 120 p., Dissertação (mestre em Tecnologia de Alimentos), Departamento de Tecnologia de Alimentos. Universidade Federal do Ceará (UFC).

[10] FARIA, J.B.; CARDELLO, H.M.A.B.; BOSCOLO, M.; ISIQUE, W.D.; ODELLO, L.; FRANCO, D.W. Evaluation of Brazilian woods as an alternative to oak for cachaça aging. Eur. Food. Res. Technol., v. 218, n. 1 , p. 83-87, 2003.

[11] CANAS, S.; LEANDRO, M.C.; SPRANGER, M.I; BELCHIOR, A.P. Low molecular weight organic compounds of chestnut wood (Castenea stiva L.) and corresponding aged brandies. J. Agric. Food Chem., v. 47, n. 12, p. 5.023-5.030, 1999.

[12] CHATONNET, P.; CUTZACH, I.; PONS, M.; DUBOURDIEU, D. Monitoring toasting Intensity of barrels by chromatographic: analysis of volatile compounds from toasted oak wood. J. Agric. Food Chem., v. 47, n. 10 , p. 4.310-4.318, 1999.
[13] MONEDERO, L.; OLALLA, M.; VILLALÓN, M.; GARCIALÓPEZ, H.; LÓPEZ, M.C. Standardization of the chromatic characteristics of sobretablas wine macerates obtained by an accelerated ageing technique using heating and oak shavings. Food Chem., v. 69, p. 47-54, 2000.

[14] PIGGOTT, J.R.; HUNTER, E.A.; MARGOMENOU, L. Comparison of methods of analysis of time-Intensity data: application to scotch malt whisky. Food Chem., v. 71, p. 319-326, 2000.

[15] JAGANATHAN, J. DUGAR, S.M. Authentication of straight whiskey by determination of the ratio of furfural to 5-hydroxymethyl-2-furaldehyde. J. AOAC Int., v. 82, n. 4, p. 997-1001, 1999.

[16] QUESADA, J.G.; VILLALON, M.M.; LOPEZ, G.S.H.; LOPEZ, M.M.C. Influence of aging factors on the furanic aldehyde contents of matured brandies: Aging markes. J. Agric. Food Chem., v. 44, n. 6, p.1.378-1.381, 1999.

[17] Aquino, F.W.B. Compostos fenólicos em extratos de Amburna cearensis (Fr. All.) A.C. Smith e em aguardentes no Estado do Ceará. Fortaleza, 2004, 90 p., Dissertação (mestre em Tecnologia de Alimentos), Departamento de Tecnologia de Alimentos. Universidade Federal do Ceará (UFC).

\section{6 - AGRADECIMENTOS}

Os autores agradecem à Fundação Cearense de Apoio ao Desenvolvimento Científico e Tecnológico (Funcap) pelo apoio financeiro. 